\title{
Lidil
}

Revue de linguistique et de didactique des langues

$57 \mid 2018$

Démarches créatives, détours artistiques et appropriation des langues

\section{«Les objets qui font parler » : vers une pédagogie de la création multimodale et multilingue}

Animating Objects: Towards a Pedagogy of Multimodal Creation

\section{Gabriele Budach}

\section{(2) OpenEdition}

\section{Journals}

Édition électronique

URL : http://journals.openedition.org/lidil/4922

DOI : $10.4000 /$ lidil.4922

ISSN : 1960-6052

Éditeur

UGA Éditions/Université Grenoble Alpes

Édition imprimée

ISBN : 978-2-37747-048-8

ISSN : $1146-6480$

Référence électronique

Gabriele Budach, « «Les objets qui font parler » : vers une pédagogie de la création multimodale et multilingue », Lidil [En ligne], 57 | 2018, mis en ligne le 01 mai 2018, consulté le 20 avril 2019. URL: http://journals.openedition.org/lidil/4922 ; DOI : 10.4000/lidil.4922

Ce document a été généré automatiquement le 20 avril 2019

(C) Lidil 


\title{
«Les objets qui font parler» : vers une pédagogie de la création multimodale et multilingue
}

\author{
Animating Objects: Towards a Pedagogy of Multimodal Creation
}

Gabriele Budach

\section{Introduction}

1 Ce texte présente une approche innovatrice qui vise à jumeler l'exploration d'objets matériels (Pahl \& Rowsell, 2011) et l'emploi de technologies digitales au profit de l'apprentissage de langues, d'une prise de parole critique et de l'articulation d'identités complexes (Anderson \& Macleroy, 2016). Dans ce qui suit, j'exposerai le concept et la mise en pratique d'une approche pédagogique qui, dans un premier temps, encourage l'échange autour d'objets personnels et porteurs de signification pour les apprenants afin de stimuler leur réflexion et de pénétrer des problématiques complexes. Dans un deuxième temps, les apprenants sont amenés à "mettre en scène » leur objet et à créer une histoire digitale à l'aide de logiciels disponibles pour iPad. Cette création est composée d'images, de langues, écrites et parlées, et de musique, menant ainsi à l'émergence de brefs films d'animation qui sont à la fois individuels et sémiotiquement complexes.

2 Dans l'optique de ce numéro thématique, il convient de dire qu'au sein de cette approche, l'apprentissage linguistique est conçu comme partie intégrante de l'apprentissage au sens plus large. Défini selon les termes de la sémiotique sociale, il est compris comme un processus de création de sens auquel plusieurs modes participent, tels que le verbal, le gestuel, le visuel et le tactile (Kress, 2010). Prenant au sérieux le caractère multimodal de tout apprentissage, l'approche présentée ici ne se centre donc pas sur le mode langagier d'une manière isolée ou privilégiée. Elle le considère plutôt comme un élément clé parmi d'autres dont le contenu et la forme se dessinent et se précisent au cours d'un processus 
créatif. Ce processus émerge d'un ensemble d'étapes dont certaines seront esquissées plus en détail dans ce texte.

3 En termes pédagogiques, l'approche proposée implique qu'au lieu d'exercer un contrôle relativement strict sur le choix de ressources linguistiques et leur développement, l'apprenant bénéficie d'une plus grande liberté de faire des choix autonomes qui sont en lien avec les besoins et paramètres émergents de son projet de création multimodale. Procédant ainsi, l'objectif visé en termes d'apprentissage des langues est donc de mettre à disposition de l'apprenant un cadre permettant un apprentissage actif. Selon Bransford, Brown et Cocking (2000):

Learners should be supported in taking control of, and self-regulating, their own learning. When students take ownership of the learning process and invest their identities in the outcomes of learning, the resulting understanding will be deeper than when learning is passive. (Cité par Pahl \& Rowsell, 2005, p. 147-148.)

Pour notre contexte, il est important que l'apprenant se sente motivé pour explorer son propre potentiel déjà existant et pour déterminer ses propres objectifs d'apprentissage en matière de langues qui l'aideront à articuler son histoire et son message de manière appropriée et efficace. Une telle approche a l'avantage de rendre l'apprenant responsable de son apprentissage, ce dernier étant ancré dans le contexte d'une situation de communication concrète. Cette situation a comme caractéristique d'aboutir à un produit final qui sera partagé avec un public plus grand. Cette perspective accompagne le processus et fait réfléchir le créateur de l'histoire, d'une manière plus engagée et critique, au choix de ses moyens et à la manière de raconter l'histoire.

\section{Question de recherche et ancrage théorique}

5 Le texte s'interroge, en particulier, sur la manière dont les apprenants mobilisent leur répertoire linguistique et comment ils l'intègrent dans la construction d'une histoire digitale afin de communiquer un message complexe et nuancé. Je m'intéresse à la manière dont l'apprenant représente son multilinguisme et comment il l'interprète en tant que ressource expressive lors de cette création multimodale. Il est intéressant d'observer comment les langues de l'apprenant sont mises en scène et imbriquées avec les autres modes (images, musiques ou gestes), tout en prenant une valeur symbolique bien spécifique et propre à la volonté expressive du créateur.

6 L'approche s'inscrit dans un champ de recherche plus large visant la création d'opportunités d'apprentissage authentique et le développement de littératie critique (New London Group, 1996). Inspirée par ce courant, l'approche cherche à tenir compte de l'importance des nouvelles technologies (Cope \& Kalantsis, 2000) dans la vie des jeunes de nos jours, à reconnaitre l'importance croissante du visuel (Kress \& Van Leeuwen, 1996) et à souligner le rôle de pratiques multilingues (Martin-Jones \& Jones, 2000 ; Cummins, 2006) qui représentent une ressource identitaire clé d'un nombre croissant de jeunes dans les institutions éducatives d'aujourd'hui.

7 En même temps, l'approche reconnait la multitude et la diversité des pratiques de littératies quotidiennes des apprenants. Ce terme, utilisé au pluriel, renvoie à un concept clé des New Literacy Studies et met au centre l'idée des pratiques de littératies comme la somme d'une pluralité de pratiques qui sont ancrées dans des contextes sociaux multiples. L'utilisation du pluriel est choisie pour contrer, en quelque sorte, la notion souvent très précise et monolithique qui est proposée par des conceptions 
psychologiques, se référant d'abord au contexte scolaire. Les pratiques de littératies quotidiennes sont fortement empreintes par la culture matérielle (Miller, 2010) dont le rôle a été négligé pendant longtemps, en recherche sociolinguistique (Budach, Kell \& Patrick, 2015) et en éducation (Pahl \& Rowsell, 2011) ou en didactique des langues. S'inscrivant dans le material turn (Bowker \& Star, 1999 ; Star, 2010), cette recherche vise donc à revaloriser la matérialité et à reconnaitre son potentiel comme une ressource didactique valable.

Misant sur le potentiel d'objets, l'étude cherche à tirer profit de deux capacités d'objets décrites par la philosophie phénoménologique et, plus récemment, en New Literacy Studies (Kell, 2009). Merleau-Ponty (1964) reconnait aux objets la fonction de dépositoire de signification, aptes à accumuler de la mémoire historique et culturelle. Les objets représentent donc des réservoirs d'histoires et de savoirs qui peuvent être actualisés et articulés, entièrement ou partiellement, dépendamment de l'expérience et des connaissances de ceux et celles qui participent à l'échange autour de ces mêmes objets. Deuxièmement, les objets jouent un rôle clé en tant que lien, chainon ou interface et aident à la création de relations humaines, à la co-construction du savoir et à l'échange de différentes interprétations du monde, matériel et symbolique, qui circulent, sous forme de significations différenciées, à travers le temps et l'espace (Kell, 2009).

Plus récemment, les recherches en littératies d'artefacts (Pahl \& Rowsell, 2010) ont montré que le travail autour d'objets peut produire des effets très fructueux, notamment dans des contextes pédagogiques. Il incite à développer l'articulation de la parole, l'envie de s'exprimer en parlant d'un objet apprécié et de partager son histoire avec d'autres. Outre cela, la conversation autour d'objets peut aider à approfondir la réflexion sur soimême et sur l'autre, tout en renforçant le sens de l'empathie et de l'appartenance (Budach, Patrick \& Mackey, 2015). Au-delà, ce travail peut sensibiliser l'apprenant à des concepts théoriques et l'aider à réfléchir à son propre positionnement face à ceux-ci.

10 L'approche proposée comporte une méthodologie souple qui peut être adaptée à différents publics et contextes d'apprentissage. Elle a à cœur de mettre en lien le vécu et l'expérience biographique avec des objectifs d'apprentissages académiques, tout en étant susceptible de tenir compte de profils sociolinguistiques, ambitions et intérêts particuliers des créateurs individuels. L'idée d'une approche de création multimodale est donc d'engendrer un espace qui permet d'explorer les possibilités de la multimodalité afin de mieux saisir ses propres ressources linguistiques et sémiotiques et de les développer de manière consciente et responsable dans la communication avec l'autre.

\section{Contexte et démarche pédagogique}

11 Les données présentées dans ce texte proviennent d'un contexte d'enseignement universitaire et ont été collectées lors d'une école d'été, organisée du 18 au 23 septembre 2016, à l'université du Luxembourg par le programme du Master en communication et apprentissage en contextes multilingues et multiculturels (Master in communication and learning in multilingual and multicultural contexts). Les participants - au total 16 - étaient, en majorité, des étudiants de ce programme, en plus d'un étudiant du Bachelor en sciences de l'éducation, un doctorant et un post-doctorant. L'école d'été était intitulée « Exploring digital creation: Multilingual identities in the making " et misait d'abord sur l'exploration d'identités linguistiques et culturelles. Ce thème est un phénomène de société marquant, aussi bien qu'une préoccupation théorique et expérientielle clé dans 
notre programme de master. Notre programme est trilingue (allemand, français, anglais) et accueille des étudiants venant d'une trentaine de pays divers dont la majorité a une langue première différente des trois langues d'instruction de notre programme.

En préparation de l'école d'été, tous les étudiants avaient reçu la même consigne qui consistait en deux parties : (1) relire le texte de Mary Louise Pratt (1991) sur «L'art de la zone de contact » (Arts of the Contact Zone) (la majorité des participants était familière avec ce texte, car il avait été traité dans d'autres contextes de cours du programme de master) ; (2) choisir un objet qui, pour eux, représenterait une zone de contact. Selon Pratt, celle-ci est définie comme un espace dans lequel plusieurs cultures se rencontrent et se mélangent, ce qui peut produire des échanges plutôt harmonieux ou conflictuels. Il était suggéré que le choix d'objet devait avoir un lien avec une expérience personnelle du participant et que l'objet et son histoire devaient être présentés aux autres participants le premier jour de l'école d'été. Ce moment de partage était le point de départ commun à partir duquel les mises en scène d'objets commencèrent à se dessiner. Le processus créatif était aidé par deux réalisatrices et artistes digitales, Bo Chapman et Zoé Flynn, de Salmagundi Films à Londres, qui étaient à disposition pour encadrer les étudiants et pour les accompagner dans la découverte et la maitrise des outils digitaux. Tous les participants avaient choisi de développer des projets individuels et de travailler avec l'application iStopMotion. En même temps, la coopération, l'entraide et l'inspiration mutuelle étaient une force motrice importante partagée tout au long du processus. En étape finale, le dernier jour de l'école d'été, les films de tous les participants ont été projetés, chaque projection étant précédée d'une introduction par le créateur.

Conceptuellement, cette démarche reflète les principes pédagogiques de la multilittératie établis par le New London Group (1996), son objectif ultime étant défini comme suit :

The essence of this framework is that students should be given opportunities to engage in meaningful experience and practice within a learning community, and explicit instruction should support the development of concepts and understanding as required. Students should also have opportunities to step back from what they have learned and examine concepts and ideas critically in relation to their social relevance. Finally, they should be given opportunities to take the knowledge they have gained further to put it into play in the world of ideas and come to understand how their insights can exert an impact on people and issues in the real world. (Cité par Cummins, 2006, p. 54.)

Selon ce cadre, on distingue trois phases qui permettent de (1) créer un cadre explicatif pour l'activité (overt instruction), (2) engager la réflexion critique sur des problèmes sociaux réels qui sont mis en lien avec le vécu des étudiants (critical framing), pour enfin (3) mener à la transposition de l'expérience d'apprentissage à d'autres contextes, ce qui représente chez l'apprenant une transformation au bénéfice d'une compréhension et réflexion approfondie (transformed practice).

Dans notre scénario, le partage du concept de «zone de contact » représente le point de départ commun permettant une cohérence entre les projets à créer (overt instruction). Un premier moment d'encadrement critique évolue lorsque les étudiants sont amenés à choisir un objet qui, pour eux, représente une zone de contact. À travers ce processus, ils mettent en lien le concept avec leur vécu et expérience biographique (criticial framing). En même temps, ils interprètent le concept en lui donnant une forme matérielle distincte et en choisissant un modèle de représentation parmi les représentations déjà existantes au monde. Selon Sullivan (2017) qui se réfère à Kress (2010), l'apprentissage repose sur deux principes qui sont à l'œuvre lorsqu'un objet (ou modèle) est au centre de l'activité d'apprentissage : 
Students learn in two ways with modal representations-first, they learn by interpreting and internalizing the meaning of the sign; second they learn by producing modal representations. (Sullivan, 2017, p. 17) cette étude, est fortement engagée au moment où l'apprenant choisit l'objet et son histoire en lien avec un moment biographique précis, tandis que la production d'une nouvelle représentation modale est mise en œuvre lors de l'étape suivante - la création de l'histoire digitale. C'est à travers cet acte qu'un nouveau dessin est mis au monde, engendrant pour l'apprenant une pratique transformatrice. Suivant l'inspiration des études sur la nouvelle matérialité (new materialism), il convient de dire que l'objet peut prendre un rôle actif dans la construction du sens (Bowker \& Star, 1999; Star, 2010) et que souvent c'est l'objet, sa forme, ses propriétés matérielles et esthétiques, qui suggèrent une certaine interprétation plutôt qu'une autre. La création de nouveaux signes ne repose donc pas uniquement sur un acte subjectif du créateur, mais se nourrit d'une co-construction à laquelle participent la personne et l'objet à parts égales. Au même titre, la compréhension du concept de «zone de contact » est approfondie et de nouvelles connaissances de technologies digitales sont acquises par les étudiants lors de cette deuxième phase.

\section{Analyse de données : trois mises en scènes du multilinguisme}

17 L'analyse portera sur trois participants de l'école d'été et leur processus de création. Chacune des créations puise dans un ensemble de ressources sémiotiques qui sont à la portée et disposition de la personne créatrice et représente la mise en scène d'un répertoire multilingue bien particulier. La configuration et la mise en forme précise de chacun des produits finaux émergent peu à peu lors du processus de la création multimodale. Celui-ci fait apparaitre, à travers le choix de ressources linguistiques (écrites ou orales) et d'autres modes, des images ou sons musicaux, un assemblage de texture unique. Dans les trois cas examinés ici, nous découvrons des mises en valeur du multilinguisme bien particulières qui co-construisent la signification de l'objet en zone de contact et témoignent de la personnalité de la créatrice, de son identité et de sa volonté de raconter l'histoire et de la faire vivre.

18 La première des créatrices, Elena, est d'origine russe et apprend l'anglais, l'allemand, le français et depuis récemment le luxembourgeois. Pour sa création filmique, elle explore la trajectoire d'un texte littéraire russe célèbre, Crime et Châtiment de Fiodor Dostoïevski, et sa traduction et réception en plusieurs langues. La deuxième créatrice, Raluca, est d'origine roumaine et dispose, grâce à son parcours biographique, d'une identité culturelle et linguistique complexe composée d'éléments roumain, irlandais, belge et luxembourgeois. Pour son projet d'histoire digitale, elle décide d'explorer sa collection de blouses folkloriques roumaines. La troisième créatrice, Paula, est de langue maternelle polonaise, parle l'anglais, le français et quelques mots d'allemand, d'espagnol et de chinois. Pour son projet, elle choisit un rosaire tibétain qu'elle explore en tant qu'outil de prière et objet symbolique à portée universelle.

19 Par la suite, je m'intéresserai à trois moments du processus de création. En premier, je tiendrai compte de la photo et du texte que les participants devaient soumettre avant le début de l'école d'été. Je les examinerai afin de saisir leur idée et l'état de leur

Lidil, 57 | 2018 
développement au début de l'école d'été. Ensuite, j'inviterai le lecteur à regarder les trois films créés par les trois réalisatrices qui sont disponibles sur Vimeo ${ }^{1}$. Enfin, je traiterai les commentaires fournis par les trois participantes sur leur expérience de création d'histoire digitale après l'école d'été, lors d'une discussion de groupe et sous forme d'une réflexion personnelle écrite, afin de comprendre l'impact du projet sur leur personne et apprentissage.

\subsection{Un livre qui fait découvrir plusieurs lectures du monde}

Le texte qu'Elena propose se lit comme suit:

The book Crime and Punishment by F. M. Dostoevsky is one of the greatest cultural assets of Russia and everyone in my country have already read it or will do it because it is an obligatory book for reading in schools. Also this book was translated into several languages and became widely known in Europe in 19th century. During my living in Luxembourg, I found out that even now this novel is very popular among young generation. Certainly, it was very exciting to discuss this book with people, who have another cultural background, because their perception and interpretation focuses on different elements, events and details, which were neutral for me. Such kind of experience made me reread the book in English in order to refresh my perception and to find "new colours" of the novel.

Image 1. - L'objet d'Elena.

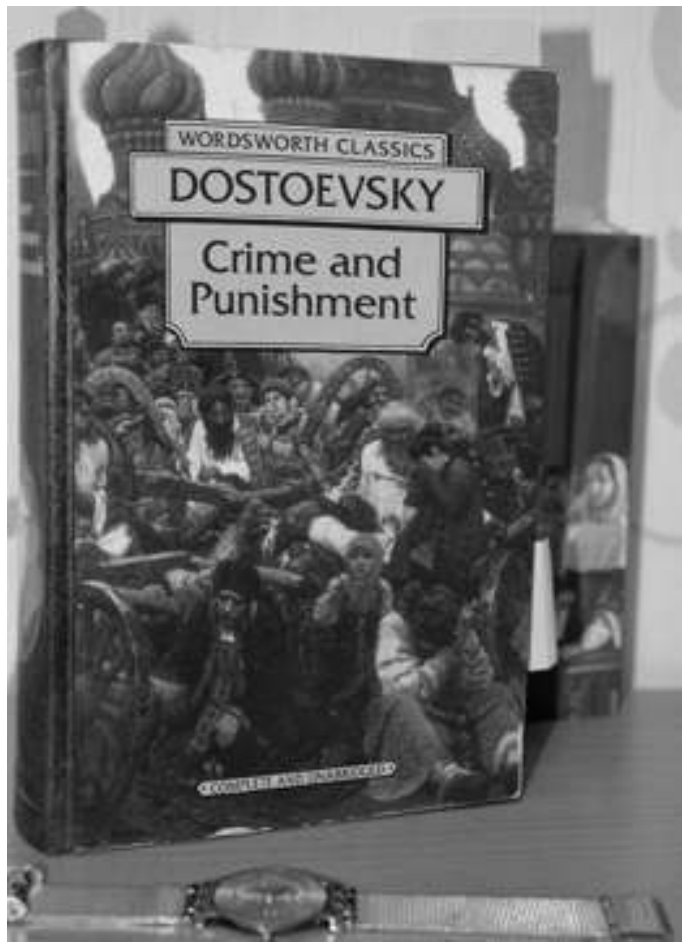

Dans ce texte, relativement court, Elena établit la valeur et le statut de l'œuvre en tant que texte clé de la littérature classique russe et élément obligatoire du curriculum national en Russie. Elle est consciente de son influence au $\mathrm{XIX}^{\mathrm{e}}$ siècle lorsque, après sa parution, il a été traduit dans bien d'autres langues. Pourtant, elle se montre surprise du fait que de jeunes Luxembourgeois, rencontrés depuis son arrivée au Luxembourg, le connaissent et s'y identifient également. À sa grande surprise, l'expérience de vivre au Luxembourg lui a apporté l'opportunité de redécouvrir une œuvre qu'elle connaissait depuis longtemps et de la relire dans une autre langue, notamment en anglais. Cette 
expérience lui apprend à apprécier l'œuvre, selon un nouvel éclairage, et de reconnaitre son pouvoir d'engager et d'enrichir le dialogue interculturel. Pour Elena, c'est donc un livre qui résume son expérience récente de la «zone de contact» et qui lui permet, à travers cet objet bien connu, de se redécouvrir elle-même miroitée dans la perception de l'autre. La façon dont elle met en scène l'objet peut être observée dans son film².

Rappelons que selon Kress (2010) chaque film peut être considéré comme la création d'un nouveau modèle de représentation. Le processus de chaque création profite des possibilités modales (affordances) de l'animation, le terme se référant aux potentialités et contraintes de modes différents et leur capacité (ou incapacité) d'exprimer, de représenter ou de communiquer des contenus particuliers (Kress, 2010). Par exemple, la danse offre des possibilités différentes du chant ou de la peinture. Dans sa création, Elena réussit à créer un objet multilingue capable de se transformer et de changer de langue, passant du russe à l'anglais. Cette métamorphose est mise en scène à travers l'image d'une boule de papier froissé qui se forme d'abord de pages écrites en russe. La boule ensuite disparait du champ visuel et, peu de temps après, réapparait, cette fois-ci composée de pages écrites en anglais, qui se dévoilent lorsque la boule se transforme, à nouveau, en feuilles de papier lisses.

Pour rendre l'idée du livre multilingue, Elena ajoute un élément de travail collaboratif et demande aux autres participants de lui fournir leurs citations préférées de cette œuvre. Ces citations sont représentées dans la deuxième partie du film. Ainsi, elle arrive à intégrer et visualiser l'idée que d'autres langues (notamment l'allemand) participent également à l'univers discursif dans lequel l'œuvre circule. Profitant de la magie de l'animation, le livre est mis en scène comme une source d'inspiration et invitation à l'élévation de soi pour le spectateur - mise en mots par la citation « find yourself in a hero ». Par ailleurs, le pouvoir du texte est amplifié par la taille du livre, paraissant très grand et majestueux face à l'homme qui est de proportion minuscule et qui semble emballé par la présence de l'objet et attiré pour s'y immerger et le découvrir en profondeur.

En termes de langues, le russe et l'anglais jouent les rôles principaux dans le film. L'anglais représente une langue seconde pour Elena et la langue commune des étudiants du programme. Elle a donc une fonction double qui est (1) de transcrire l'expérience de la créatrice comme apprenante de l'anglais et de la culture anglaise et (2) de faire le lien avec le public qu'elle s'imagine majoritairement anglophone. L'anglais apparait d'abord sur la couverture pour permettre au public d'identifier le titre du livre, tandis que l'histoire sous forme de corps textuel est d'abord explorée en russe. On voit un stylo, qui semble bouger sans intervention humaine, marquer des passages en russe avant que l'acte de transformation de l'objet ait lieu, ce qui permet ensuite la relecture du texte en langue anglaise. Ainsi, le film reprend l'expérience biographique d'Elena, qui a d'abord étudié le texte en russe avant de le découvrir en anglais.

Les remarques suivantes, qu'elle formule à propos de son projet après l'école d'été, nous permettent de saisir un développement de l'apprentissage qui émerge de son travail de projet.

This book is an object of the contact zone, because it is known in the world even nowadays, it means that people from different cultures read and interpret the novel in accordance with their different cultural backgrounds. The book connects people from all over the world but stays very personal for everyone. With this story I want to show that every book opens for you its world, but the same book in another language demonstrates you its world from another perspective. 
Si l'on compare avec son texte écrit avant l'école d'été, il s'avère que maintenant elle nomme expressis verbis le concept de "zone de contact», ce qui renvoie à une réflexion plus approfondie par rapport au terme. En outre, elle reconnait le potentiel d'un objet, notamment d'un texte littéraire du xix ${ }^{e}$ siècle, à fonctionner comme un lien ou chainon permettant aux gens d'entrer en contact, d'échanger et de se rapprocher à travers le dialogue. La manière dont elle formule ses propos et l'usage de termes plus précis (en anglais) indique un avancement à deux niveaux: par rapport à sa compréhension du concept de zone de contact et au rôle que peuvent y jouer des objets, d'une part, et par rapport à sa capacité à exprimer ses idées en langue anglaise, d'autre part. Sur le plan des idées, elle reconnait donc à un texte littéraire de circulation globale la capacité de servir d'intermédiaire au sein d'un dialogue interculturel dans lequel différentes positions sont possibles et légitimes. Une telle vision transcende l'idée d'une seule lecture juste, de même que le cadre de discours nationaux promouvant souvent une lecture et interprétation monodimensionnelle.

\subsection{Une chemise brodée qui protège le corps}

Le texte de Raluca se lit comme suit :

It is a Romanian blouse and I wear it in many instances when I need protection. I was not in touch with my Romanian side for a long time. Ever since I left Romania, I considered myself a citizen of this world, a European if I have to narrow down the geographical area. But when I arrived in Luxembourg my identity was put to question and examined and there were people keen to find out where I come from. I myself asked this question so many times: how do I define myself? My name is Turkish, I was born in Ireland, but I was raised and educated in Romania, I went to Ireland to continue my education and build a career. I got married and came to Luxembourg, but I actual only work in Luxembourg, and I live in Belgium. So many places and instances, and they are bound to have a small or a large influence on me. It was that time when I needed a core, I have many layers, but what is my core? I am not Turkish, I am not Irish, I am not Luxembourgish and I'm definitely not Belgian, I am Romanian. And what better object to protect my core if not a Romanian blouse? 
Image 2. - Les objets de Raluca.

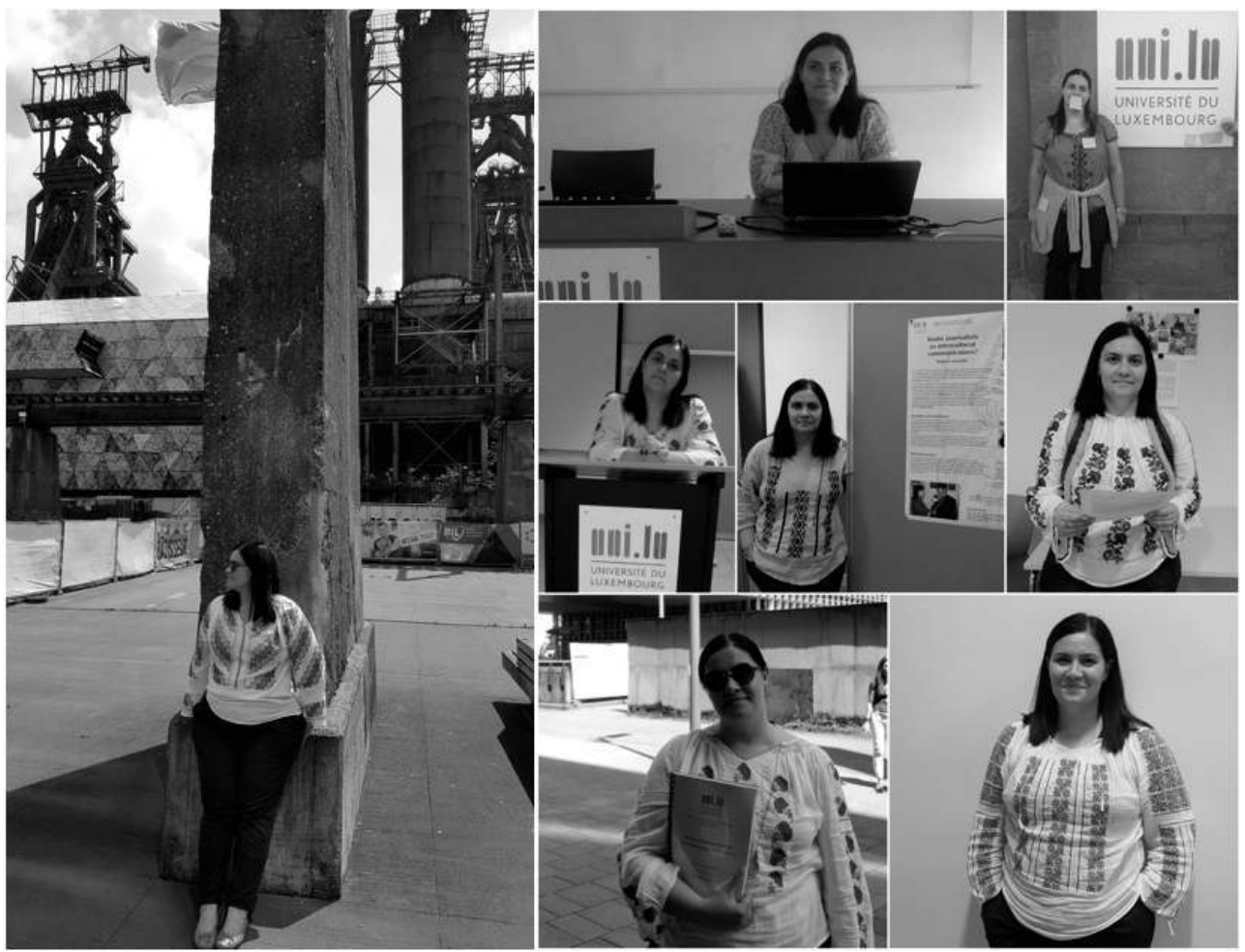

(Suite du texte de Raluca)

The Romanian blouse is part of the traditional folk Romanian costume. It is hand made from the fabric to the embroidery to the tides that put all together. Typically a woman would work on her Romanian blouse for half of a year, the Winter half, when there were no works on the field. So the Romanian blouse was done during the wintertime. Women would gather together to create their blouses, but they were also sewed in secret, because the woman wanted her blouse to be unique and special and she didn't want anyone to copy her patterns. That is why there are no two Romanian blouses alike. The most distinctive feature of a Romanian blouse is the sleeve. There, the woman would embroider her identity, where she came from, her hopes for the future and where she would end. The sleeve with its patterns would carry the story of the woman who made the blouse over centuries. In our times, the old blouses are part of museums and women of this time are gathered in groups trying to decipher the symbols sewed on the old blouses. The blouse itself evolved (or devolved) and now the blouses are not made for celebrations, but for everyday life. Mystery was replaced by comfort, but still the mission of the Romanian blouse is carried on. When I wear my Romanian blouses I am not just a face in the crowd, I stand out, I say where I am from and where I'm going. I also feel protected. I was never in danger and I was never alone when I wore a Romanian Blouse, because I carry with me the symbols of my people. So to answer my initial question, I am a citizen of the world wearing a Romanian blouse :) To the summer school I will bring a Romanian blouse from the present, but also an old one and I would tell you its story, because all Romanian blouses have stores. In the picture attached is me wearing a Romanian blouse for almost all the presentations and important moments of the Master, including my defense when again I was wearing a Romanian blouse.

Raluca soumet un texte qui est beaucoup plus long et détaillé que celui d'Elena. En fait, l'avènement de l'école d'été lui permet de creuser davantage un projet de recherche identitaire qu'elle poursuit depuis quelque temps et au sein duquel les blouses traditionnelles roumaines ont pris une place centrale. Le texte qu'elle propose semble remplir deux missions : (1) il traite de sa quête identitaire qui émerge d'un parcours de 
migration complexe durant lequel son identité est interpellée par les gens de son entourage ; (2) elle y déploie une grande connaissance par rapport à l'histoire des blouses roumaines en tant qu'objet d'identification féminine. De plus, elle explique de manière détaillée les origines et usages variés de la blouse en tant qu'objet festif, secret, ou muséal, adoptant une posture quasi documentaire. La manière dont elle met en scène sa collection d'objets peut être découvert dans le film de Raluca ${ }^{3}$.

Si l'on compare cette mise en scène et ce modèle de représentation (Kress, 2010) avec ceux créés par Elena, nous constatons des similitudes, mais aussi des spécificités importantes. Les deux projets sont fortement personnels et ils se distinguent de manière frappante, notamment par rapport au choix de moyens expressifs et esthétiques. Elena construit son message en exploitant la magie d'objets animés et en renonçant à la parole parlée au profit de la représentation de l'écrit ; tandis que Raluca met en valeur beaucoup plus la parole parlée et sa propre voix. Elle reste fidèle à l'idée d'un style journalistique et documentaire, ce qui reflète son expérience professionnelle et son expertise en tant que journaliste qui travaille pour la radio au Luxembourg.

Une grande partie du texte est présentée en anglais, qui est aussi la langue comprise par la majorité des étudiants en master. Il faut noter que la créatrice conserve, en grande partie, la version initiale du texte. En fait, à cause de sa longueur, elle a dû le réduire de quelques paragraphes pour laisser plus d'espace à d'autres modes de composition, notamment le visuel. Pendant qu'Elena se sert de l'écriture en différentes langues, Raluca mise sur la parole parlée, notamment en anglais et roumain. Quant au roumain, langue première de Raluca, son emploi est tout à fait remarquable. Elle reprend une partie bien particulière de son texte original en anglais qui traite de la manche de la blouse.

The most distinctive feature of a Romanian blouse is the sleeve. There, the woman would embroider her identity, where she came from, her hopes for the future and where she would end. The sleeve with its patterns would carry the story of the woman who made the blouse over centuries.

31 Dans le film, cette partie du texte est remplacée par une description de la manche en langue roumaine qui introduit des termes utilisés en broderie. À mon avis, ce choix est significatif à deux niveaux. Il donne une place à la langue roumaine qui représente une source d'identification importante pour la créatrice. Parallèlement, l'endroit semble choisi avec soin, car la manche, dans la fabrication artisanale d'autrefois, représentait la partie la plus intime de la blouse portant un message très personnel de la couturière qu'elle était soucieuse de garder secret. Afin d'assurer la compréhension par le spectateur que la créatrice n'imagine pas être romanophone, elle profite de la multimodalité et de la possibilité de créer du sens à travers différents modes. Dans cette partie, elle se sert de l'application ProCreate pour attirer l'attention sur les différentes parties brodées de la manche qui sont, à la fois, encerclées et nommées avec leurs termes techniques de broderie en roumain, ce qui crée presque l'impression d'une leçon virtuelle en broderie avec sa terminologie. En résumé de son expérience, Raluca écrit dans une réflexion personnelle après l'école d'été :

When I was asked to create a story around an object, I knew exactly which object to pick, a Romanian blouse. I discovered them about four or five years ago when I started asking questions about Romania and when I wanted to find things that would connect me to that side of my identity. My movie was meant to tell the story of my object, the Romanian blouse, but in the process, it also told my own identity story.

D'abord elle explique sa découverte de la blouse roumaine qui, pour elle, s'est produite en vivant loin de son pays natal. Ensuite elle remarque que, pour elle, le processus de la 
création du film lui a permis de jumeler deux fils conducteurs de l'histoire, celui de sa propre recherche identitaire et celui de l'histoire de l'objet qui auparavant avaient pour elle une existence plutôt séparée l'une de l'autre. L'expérience lui a donc permis de prendre sa place dans la lignée de générations de femmes roumaines, pour qui la blouse représente un objet d'identification forte. Elle lui a permis aussi de se sentir plus ancrée dans cette communauté qui, de nos jours, est de plus en plus transnationale. Même si, à première vue, l'apprentissage des langues n'est pas au cœur de la préoccupation de la créatrice, le fait de pouvoir articuler les langues attachées à son cheminement biographique et identitaire complexe lui donne un sentiment de confiance et de pouvoir renouvelé.

À la question « Did the experience change you? If so, in what way? ", elle répond :

I think the whole experience changed me and it gave me more confidence in my powers. After just one week I had to present my movie around my idea and I think I did a good job. Also, my movie was about my own identity and it raised a lot of questions inside about that subject, questions that I got answers for during the week.

\subsection{Des perles sur un fil}

\section{Image 3. - L'objet de Paula.}

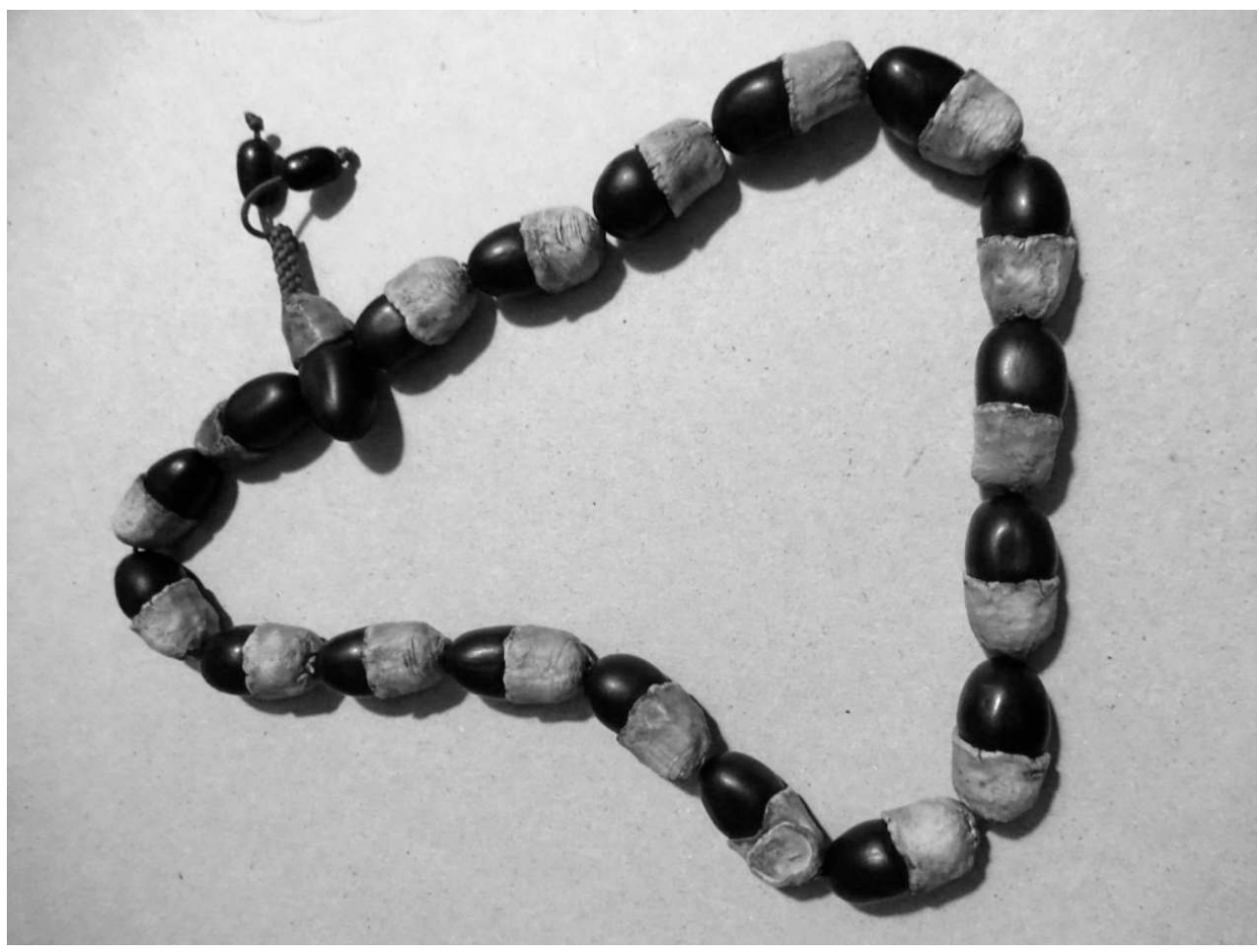

Le texte proposé par Paula se lit comme suit :

The object which I present is a Tibetan device for praying. I bought it being sure that it was a piece of jewelry. Later on I was told about its inherent purpose. This encounter made me think about [...] the form of objects and how it influences our perception and the ways in which objects are used. Taking as an example the rosary, its form is limited to the [pearls] on a line. It seems that the shape, its length and size allows and determines the particular action to be taken.

In the context of rosary, it means that the beads should fit into a hand and [...] allow the smooth movement of the hand [...] [with] the bullets go[ing] round in circles. Finally, it is a 
[...] cultural spiritual practice of praying. Moreover, this action functions as a transcultural experience in the sense that in different religions the praying devices are similar in form and shape. From that point, I can conclude that the form makes us understand the purpose of an object, but our understanding is determined by our [previous] experience with this form [ and object]. [...] Thus, in the context of contact zones, I believe it is the purpose of action to bring out their particular meaning. However, my question in the context of the contact zone is; to what extent does such practice help people to understand that even across the globe their cultures do have similar meanings, rather than fundamentally different ones? In the context of my object, I wonder how and why did it happen that the Tibetan and Christian rosary have a similar shape and purpose.

35 Avec son choix d'objet, Paula explore un objet qu'elle avait acheté lors d'un séjour en Chine et qu'elle prenait d'abord pour un collier - bijou ou objet de décor. C'est en conversant avec une passante qu'elle a pu découvrir que la vraie destination de l'objet était celle d'un rosaire - objet de prière et pratique religieuse. Ce qui l'intéresse, par la suite, c'est d'explorer le lien entre la forme d'un objet, sa fonction, son emploi et ses significations possibles dans différentes cultures. Elle s'interroge sur la possibilité de deviner la fonction d'un objet à partir de sa forme et des actions qu'il permet d'exécuter. Elle porte donc un intérêt particulier à l'objet, sa matérialité et ses possibilités d'usage. À travers cette focalisation, elle réussit à relever à la fois, la signification culturellement spécifique et celle plus universelle et liée à la pratique religieuse de prière dans différentes religions. Cette mise en balance permet de voir le rosaire plutôt comme un pont dans l'échange entre les cultures, au lieu d'un objet qui crée obstacle et renforce la différence. La façon dont elle met en scène l'objet peut être observée dans le film de Paula ${ }^{4}$.

On découvre une œuvre dans laquelle Paula fait valoir son expérience et expertise d'artiste. Elle est diplômée de l'Académie des beaux-arts en Pologne et a élargi son expérience par des stages de peinture en France et de calligraphie en Chine. Dans son film, elle explore la matérialité et la forme du rosaire par différentes approches. Après la présentation de son objet, d'autres participants proposent de lui amener des rosaires qu'on voit tous jumelés dans la première scène du film. Ensuite, on la voit peindre la forme d'un cercle avec sa main. Enfin, elle explore son objet en le faisant glisser dans sa main. Cette exploration tactile est accompagnée de sons (en musique et mots en différentes langues) qui suggèrent un contexte d'utilisation de l'objet pour la prière, donc une pratique religieuse. L'origine de l'objet est soulignée par une musique qui ressemble au chant de prière bouddhiste. Le lien avec sa propre biographie et socialisation dans la foi catholique en Pologne est relevé par des bouts de prière qu'elle récite à voix basse en langue polonaise. Cette utilisation de voix en plusieurs langues est particulièrement intéressante. On a vu que Raluca préfère un ton plutôt déclamatoire pour mettre en scène son récit biographique, tandis que Paula choisit une manière très discrète d'utiliser la voix humaine qui sert à créer le contexte et l'ambiance dans lesquels l'objet reçoit sa signification comme outil rituel. La juxtaposition des contextes sonores et linguistiques, avec des images de rosaires qui restent stables, suggère qu'il s'agit d'un objet transculturel et d'une pratique religieuse qui transcende les religions, régions et cultures du monde. L'objet devient donc l'intermédiaire d'un message qui souligne l'idée de l'unité dans la diversité et résiste, en quelque sorte, à la prise de possession d'un objet aux fins d'un discours ou projet nationaliste. Ce message reconnait enfin que la culture matérielle souvent unifie les personnes de différentes cultures beaucoup plus qu'elle ne les sépare. Pour ce qui est de l'écrit, Paula s'en sert de manière très économe. Seul le titre apparait 
en écriture et se lit "focus is one form is one ", ce qui résume l'idée clé de son projet, à savoir la recherche des liens entre formes et significations d'objets.

Essayons de faire le point sur l'expérience de ces trois créations multimodales. En résumé, on peut retenir le constat qui émerge lors d'une discussion de groupe suite à l'école d'été, quand Sammy, un des participants, propose :

From the Summer School I will take away that everyone has an identity [...] and that the identity is very individual. And everyone has something inside of him or her that can't be seen by others, but that can be opened up by objects. So, objects help to discover the hidden identities.

Paula est d'accord et renchérit :

I think that is a very good point of the hidden identities revealed by objects. This is what I understood by analyzing my own object focusing on the shape [...] I found out that it is the form of a circle finally that has a great meaning [...] for us humans [...] so I tried to stick to that shape and to develop it.

\section{Conclusion}

Nous retenons qu'avec une même consigne, chaque étudiant a développé un projet bien différent des autres. D'un côté, nous avons vu émerger des modèles de représentation de la «zone de contact» très individuels, mais né chacun du processus de la création multimodale et des répertoires linguistiques, des expériences et talents de leur créateur. D'un autre côté, nous avons pu observer qu'à travers ces créations, un message plus global s'est manifesté qui transcende l'histoire biographique de son créateur. Dans chaque cas, les répertoires multilingues et multiculturels ont joué un rôle clé dans la composition du message. Il s'avère que la langue première semble matérialiser le lien biographique personnel, tandis que l'anglais ou d'autres langues construisent la dimension plus universelle du message. Elena nous fait découvrir d'abord le livre en russe qui, ensuite, se transforme en version anglaise. À travers cette transformation, elle invite le spectateur à participer à la découverte de nouvelles lectures et interprétations du monde, tous prenant leur source dans le même texte. Raluca réserve la langue roumaine à l'explication de la broderie de la partie la plus secrète du vêtement. Elle réussit, comme elle dit, à jumeler à la fois son projet de recherche identitaire personnelle et la mise en lumière d'un objet, de son histoire, de sa fabrication et de sa signification particulière qui sont chères à la créatrice et qu'elle aime partager avec d'autres. Paula met en scène la langue polonaise pour représenter la pratique de la prière avec laquelle elle a grandi dans la religion catholique. L'usage d'autres langues dessine un contexte plus large qui met en lumière, dans son cas, la transcendance symbolique du rosaire et celle de la forme du cercle. C'est elle qui, dans l'exploration de l'objet, porte un intérêt prépondérant à la matérialité, la forme et la fonction de l'objet dont la signification ne se découvre que graduellement et à travers l'exploration multisensorielle.

Malgré la spécificité de chaque projet, les langues - maternelle et autre(s) - aident à articuler une double appartenance identitaire. À Elena, elles permettent de s'affilier à la culture et tradition littéraire russe et à se faire une place dans une communauté de lecteurs transnationale. Pour Raluca, le roumain crée un lien privilégié avec des femmes roumaines dont elle partage l'héritage, le savoir et le respect pour le travail de chaque créatrice, tandis que l'anglais donne une voix à son appartenance à la communauté des « citoyens du monde». Enfin, pour Paula, le polonais crée un point d'ancrage dans son 
vécu biographique et savoir symbolique acquis lors de son enfance, qui est mis en lien avec d'autres types d'expériences culturelles afin d'aller au fond de questions philosophiques qui cherchent à comprendre la signification des choses et comment elles naissent dans différents contextes.

Dans tous les projets, les langues jouent un rôle central. Pourtant, la valeur de la création et le gain pour les participants en termes d'expérience et d'apprentissage se mesurent mal lorsqu'on ne juge que le produit final. À tous les participants, c'est le processus qui a apporté une expérience appréciée, même s'il est peu repérable aux traits directement observables du film. Le processus a amené à un approfondissement de la réflexion tout en nourrissant le plaisir de découvrir et d'évoluer, chacun pour soi-même et à côté de l'autre. En résumé, voici ce que Paula partage avec nous :

I believe it was incredible work [...] and we influenced each other, the energy was there [...] we were a group but the projects were very individual [...] this is probably why I used painting because I wanted to show my identity as a painter [...] a tool that is so close to me.

\section{BIBLIOGRAPHIE}

ANDERSON, Jim \& MACLEROY, Vicky (dir.). (2016). Multilingual Digital Storytelling: Engaging Creatively and Critically with Literacy. Londres : Routledge.

BOWKER, Geoffrey \& STAR, Susan Leigh. (1999). Sorting Things Out. Classification and Its Consequences. Cambridge : MIT Press.

BRANSFORD, John D., BRown, Ann L. \& CoCKIng, Rodney R. (dir.). (2000). How People Learn: Brain, Mind, Experience, and School. Disponible en ligne sur <http://www.colorado.edu/MCDB/ LearningBiology/readings/How-people-learn.pdf> (consulté le 3 juillet 2017).

Budach, Gabriele, Kell, Catherine \& PATRICK, Donna. (2015). Objects and Language in Transcontextual Communication. Social Semiotics, 25(4), 387-400.

BUDACH, Gabriele, PATRICK, Donna \& MACKAY, Teevi. (2015). “Talk around Objects”: Designing Trajectories of Belonging in an Urban Inuit Community. Social Semiotics, 25(4), 446-464.

CoPE, Bill \& KalAnTZIS, Mary. (2000). Multiliteracies: Literacy Learning and the Design of Social Futures. Psychology Press.

Cummins, Jim. (2006). Identity Texts. The Imaginative Construction of Self through Multilingual Pedagogy. Dans O. García, T. Skutnabb-Kangas \& M. Torres-Guzmán (dir.), Imaging Multilingual Schools. Languages in Education and Glocalization (p. 51-68). Clevedon : Multilingual Matters.

Kell, Catherine. (2009). Literacy Practices, Text/s and Meaning Making across Time and Space. Dans M. Prinsloo \& M. Baynham, The Future of Literacy Studies (p. 75-99). UK : Palgrave Macmillan.

KRESS, Gunther. (2010). Multimodality. A Social-Semiotic Approach to Contemporary Communication. Londres : Routledge Falmer.

KRESS, Gunther \& VAN LEEUWEN, Theo. (1996). Reading Images: The Grammar of Visual Design. Psychology Press. 
MARTIn-Jones, Marilyn \& JonEs, Kathryn. (2000). Multiliteracies. Amsterdam : John Benjamins.

MerleAu-Ponty, Maurice. (1964). Les sciences de l'homme et la phénoménologie. Bulletin de psychologie, 18(3-6), 141-170.

MiLleR, Daniel. (2010). Stuff. Cambridge : Polity.

PAHL, Kate \& RowSELL, Jennifer. (2005). Literacy and Education: Understanding the New Literacy Studies in the Classroom. Londres : Sage.

PAHL, Kate \& RowSELL, Jennifer. (2011). Artifactual Critical Literacy: A New Perspective for Literacy Education. Berkeley Review of Education, 2(2), 129-152.

PRATT, Mary Louise. (1991). Arts of the Contact Zone. Profession, 91, 33-40.

STAR, Susan Leigh. (2010). Ceci n'est pas un objet-frontière ! Revue d'anthropologie des connaissances, $4(1), 18-35$.

Sullivan, Florence R. (2017). Creativity, Technology, and Learning: Theory for Classroom Practice. New York : Routledge.

The New London Group. (1996). A Pedagogy of Multiliteracies: Designing Social Futures. Harvard Educational Review, 66(1), 60-93.

\section{NOTES}

1. Veuillez trouver les liens Vimeo plus bas dans le texte. L'autorisation des participants à diffuser leur travail pour des fins de recherche a été obtenue avant le début de l'école d'été.

2. <https://vimeo.com/223879666>.

3. <https://vimeo.com/223881255>.

4. <https://vimeo.com/223470527>.

\section{RÉSUMÉS}

Ce texte présente une approche pédagogique innovatrice qui vise à jumeler l'exploration d'objets matériels et l'emploi de technologies digitales. Inspiré par les travaux sur les multilittéracies et le nouveau matérialisme, le texte explore un projet de création de films d'animation autour d'objets personnels qui a été mené avec des étudiants de master lors d'une école d'été à l'université du Luxembourg. Les résultats de cette étude suggèrent que l'exploration de la culture matérielle et leur mise en scène digitale peuvent favoriser la compréhension d'idées conceptuelles complexes, telle que celle de la «zone de contact», et stimuler l'apprentissage des langues, la pensée critique et l'expression de soi.

The text presents an innovative pedagogical approach combining the exploration of material objects and the use of digital technologies. Inspired by studies in multiliteracies and new materialism, the text explores a project of animation film making around personally meaningful objects which was conducted with Master students during a Summer School at the University of Luxembourg. Results of this study suggest that exploring material culture and animating it 
through digital tools can help students to penetrate complex theoretical concepts, such as "the contact zone", and stimulate language learning, critical thinking and self-expression.

INDEX

Mots-clés : créativité, multimodalité, répertoire multilingue, objets de médiation, histoires digitales

Keywords : digital storytelling, creativity, multimodality, multilingual repertoire, artifactual literacies

\section{AUTEUR}

\section{GABRIELE BUDACH}

Université du Luxembourg 